\title{
O Eletrocardiograma de Alta Resolução no Domínio da Freqüência. Utilização de Técnicas Estatísticas de Correlação Espectral para Identificação de Pacientes com Taquicardia Ventricular Monomórfica Sustentada
}

\author{
Eduardo Corrêa Barbosa, Paulo Roberto Benchimol Barbosa, Paulo Ginefra, \\ Francisco Manes Albanesi Fo
}

Rio de Janeiro, RJ

\begin{abstract}
Objetivo - Avaliar uma melhoria da acurácia diagnóstica da taquicardia ventricular monomórfica (TVM), através do eletrocardiograma de alta resolução (ECGAR), adicionando à análise no domínio do tempo (DT), técnicas estatísticas de correlação espectral no domínio da freqüência (DF).

Métodos - Foram estudados pelo ECGAR 137 indivíduos sendo 27 com cardiopatia e TVM sustentada, 30 com cardiopatia e sem TVM e 80 sem evidências de cardiopatia. Os parametros analisados no ECGAR no DT foram: duração do QRS filtrado, RMS40 e LAS40 e, no $D F$, as médias e os devios padrões da correlação espectral intersegmentar do sinal e da banda de freqüencia delimitadora da concentração do sinal.

Resultados - A sensibilidade (Sb) e o valor preditivo positivo (VPP) do ECGAR no DT, DF e análise combinada de ambos os domínios foram, na detecção de TVM, respectivamente: $\mathrm{Sb}=59,3 \%, 63 \%$ e $81,5 \%$ e VPP $=80,0 \%$, $81,0 \%$ e $84,6 \%$.
\end{abstract}

Conclusão-A análise combinada do ECGAR nos DT e DF aumenta a acurácia diagnóstica de pacientes com apresentação clínica de TVM sustentada.

Palavras-chave: eletrocardiograma de alta resolução, taquicardia ventricular, processamento de sinal

\section{Frequency Domain Analysis of the Signal- Averaged Electrocardiogram. Application of Statistical Techniques of Spectral Correlation for Identification of Patients with Sustained Monomorphic Ventricular Tachycardia}

Purpose - To evaluate the diagnostic accuracy of monomorphic ventricular tachycardia (MVT), in patients with structural heart diseases and episodes of sustained $M V T$, using the signal-averaged ECG (SAECG) in the time (TD) and the frequency domain (FD) with statistical techniques of spectral correlation.

Methods - Twenty seven patients with at least one episode of sustained MVT, 30 patients with structural heart diseases and no evidence of ventricular arrhythmias and 80 subjects with no evidence of heart disease have been studied. SAECG was performed in all patients with the following parameters: duration of the filtered QRS, RMS 40 and LAS4O in TD and the mean and the standard deviation of both signal energy intersegmentar spectral correlation and energy frequency edge track in FD.

Results - The sensitivity(S) and positive predict value (PPV) of the SAECG in TD, in FD and combined analysis of both domains were: $S=59.3 \%, 63.0 \%, 81.5 \%$ and $P P V=$ $80.0 \%, 81.0 \%, 84.6 \%$, respectively.

Conclusion - The combined analysis of SAECG in TD and in FD improves the diagnostic accuracy in patients with S sustained MVT.

Key-words: high resolution electrocardiogram, ventricular tachycardia, signal processing
Universidade do Estado do Rio de Janeiro - UERJ

Correspondência: Eduardo Corrêa Barbosa - Av. 28 de Setembro, 77 - $2^{\circ}$ - 20551-030 - Rio de Janeiro, RJ

Recebido para publicação em 26/9/97

Aceito em 17/6/98
Nos pacientes sobreviventes ao infarto agudo do miocárdio, a presença de potenciais tardios (PT) ventriculares detectados pelo eletrocardiograma de alta resolução (ECGAR) indica área de condução lenta e fragmentada ${ }^{1,2}$.A 
identificação dos PT através do ECGAR com análise no domínio do tempo (DT) ocorre em 10\% a 50\% de várias doenças cardíacas, incluindo cardiopatia coronária, cardiomiopatias dilatada e hipertrófica e displasia arritmogênica do ventrículo direito (DAVD). O seu valor preditivo para eventos arrítmicos e morte súbita é relativamente baixo (10$30 \%$ ), embora apresentem um alto valor preditivo negativo, mostrando uma evolução livre de eventos arrítmicos em até 95\% dos casos ${ }^{3}$. A detecção de PT combinando análise nos domínios do tempo e da frequiência têm apresentado resultados controversos com alguns estudos, demonstrando melhora da acurácia preditiva ${ }^{4,5}$ e outros não ${ }^{6,7}$.

O objetivo deste estudo foi testar uma melhoria da acurácia diagnóstica de arritmias ventriculares potencialmente fatais adicionando à análise no DT as técnicas estatísticas de correlação espectral, utilizadas em nosso laboratório para estudo doECGAR no domínio da frequiência (DF), em pacientes com cardiopatias diversas e apresentação clínica de taquicardia ventricular(TV) monomórfica sustentada.

\section{Métodos}

Vinte e sete consecutivos pacientes (grupo TV) encaminhados para tratamento no Serviço de Cardiologia do Hospital Universitário Pedro Ernesto da UERJ, sendo 20 do sexo masculino, com idades variando entre 38 e 79 anos (média \pm desvio padrão $=55 \pm 8$ anos), portadores de diversas cardiopatias sendo: 17 de cardiopatia coronária (13 com infarto do miocárdio (IM) prévio), três de cardiopatia chagásica, três de cardiomiopatia dilatada, dois de DAVDe dois de cardiomiopatia hipertrófica e que sobreviveram a pelo menos um episódio clínico de TV monomórfica sustentada (duração > 30s ou necessidade de cardioversão devido comprometimento hemodinâmico) documentada através de eletrocardiograma (ECG) de 12 derivações e não associado a episódio agudo de IM, e 30 pacientes (grupo controle) acompanhados no Serviço de Cardiologia do mesmo hospital pareados, em relação ao grupo TV, para sexo, idade, tipo de cardiopatia e classe funcional (tab. I) sem antecedentes de TV foram submetidos ao ECGAR num prazo não superior a um mês do último episódio da arritmia e sem uso de medicamentos anti-arrítmicos. As drogas do grupo I foram suspensas por cinco vidas-médias e os pacientes que utilizaram amiodarona nos últimos três meses foram excluídos.

Oitenta indivíduos sem evidências clínicas de cardiopatia (grupo normal), com ECG convencional e ecocardiograma com Doppler normais e com idade média de $51 \pm 11$ anos tiveram, após consentimento, os seus ECGAR realizados para determinação dos valores de normalidade dos parâmetros estudados no DF.

Para oECGAR, utilizamos o aparelho da Art-Corazonix (ART Inc., Austin, Texas, USA) modelo Predictor II para registro das três derivações ortogonais X, Y eZ. Em média 250 batimentos foram promediados com uma frequiência de amostragem de $2.000 \mathrm{~Hz}$ até uma redução final de ruído para
0,3 $\mu$ V.Para análise no domínio do tempo, cada derivação foi tratada com filtro digital bidirecional de Butterworth de quatro pólos com cortes de 40 a $250 \mathrm{~Hz}$, conforme o protocolo do equipamento. As três derivações foram combinadas no vetor magnitude através da fórmula.

$$
\sqrt{\left(X^{2}+Y^{2}+Z^{2}\right)}
$$

Os parâmetros estudados no DT incluíram a duração do QRS filtrado (DQRS), a duração da porção terminal do QRS com amplitude $<40 \mu \mathrm{V}$ (LAS40) e a raiz média quadrática da amplitude dos 40ms finais do QRS (RMS40). A presença de PT foi definida pelo encontro de anormalidade em pelo menos dois dos três parâmetros analisados. Consideraram-se anormais as variáveis DQRS > $114 \mathrm{~ms}$, LAS40 >38ms e RMS40 $<20 \mu \mathrm{V}$ para os parâmetros de filtragem empregados ${ }^{8}$.

Para análise no DF, definimos uma área com $200 \mathrm{~ms}$ de duração, precedendo em $25 \mathrm{~ms}$ o início do QRS. O ponto inicial foi obtido através do vetor de velocidade espacial, fornecido pelo algoritmo do sistema, para evitar erro humano. O mapa tempo-freqüência foi construído dividindo a área de análise em segmentos de $25 \mathrm{~ms}$ de duração, multiplicados a uma janela do tipo Blackman-Harris e separados a intervalos de $2 \mathrm{~ms}$, e aplicando a transformada rápida de Fourier com 64 pontos de ordenação a cada segmento para determinação do conteúdo de freqüência do sinal.

O programa Predictor II da Art-Corazonix emprega para análise espectral os seguintes índices: 1) a média (CEM) e o desvio padrão (CEDP) da correlação espectral intersegmentar (CE) e 2) a média (BDM) e o desvio padrão (BDDP) da banda de freqüência delimitadora (BD) da concentração de energia do sinal. A CE analisa a distribuição da amplitude do espectro de potência do sinal em função da frequiência em que ele ocorre, estabelecendo uma correlação linear de Pearson entre consecutivos segmentos de $2 \mathrm{~ms}$ ao longo da ativação ventricular $\left(\mathrm{o} 1^{\circ} \mathrm{com} \mathrm{o} 2^{\circ}, \mathrm{o} 2^{\circ} \operatorname{com~o~}^{\circ} \mathrm{e}\right.$ assim sucessivamente). De todas as correlações realizadas são extraídas a média e o desvio padrão. Uma correlação perfeita obteria um índice de regressão linear igual a $1(\mathrm{r}=1)$ com desvio padrão de zero e representaria hipoteticamente uma condução do estímulo elétrico absolutamente uniforme. Os índices de correlação são multiplicados por 100 para facilitação de sua leitura e manipulação (fig. 1).

A BD identifica, para cada segmento analisado, qual a freqüência que delimita a concentração do sinal em $80 \%$ abaixo e $20 \%$ acima desta freqüência. De todos os segmentos analisados são extraídos a média e o desvio padrão em Hertz (fig. 2).

A análise no DF foi realizada no somatório das três derivações eletrocardiográficas ortogonais.

O programa utilizado assume a premissa de que quanto mais graves os distúrbios da condução intra-mural ventricular maiores serão os índices de CEDP, BDMe BDDP e menor o de CEM.

Para análise combinada dos domínios do tempo e freqüência, oECGAR foi considerado positivo quando encon- 

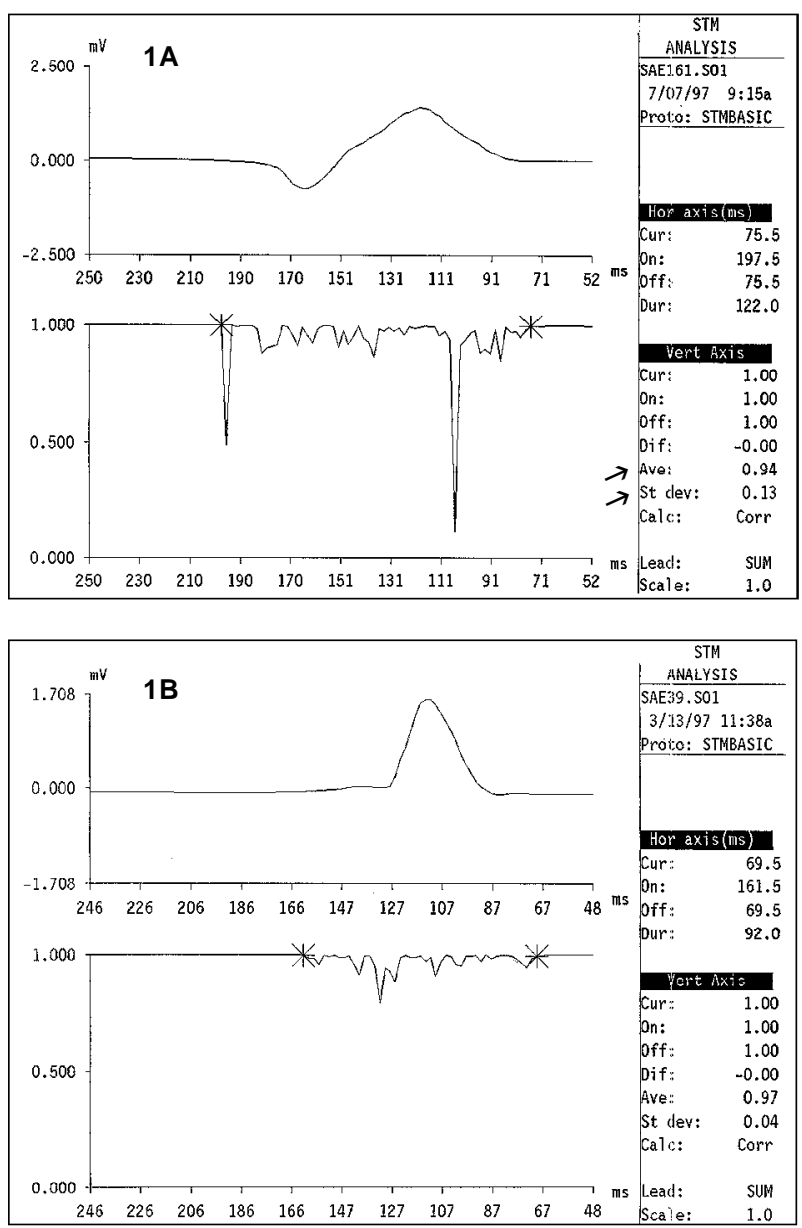

Fig. 1 - ECGAR no domínio da freqüência: correlação espectral intersegmentar (CE), média (Ave) e desvio padrão (Std) de paciente com TV (fig. 1A) e de pacientes do grupo controle (fig. 1B). Notem que em 1A, os valores de CE (seta) estão fora dos limites de normalidade.

traram-se critérios para potenciais fragmentados em pelo menos um dos dois domínios.

Os pacientes com bloqueio completo (duração de QRS $>120 \mathrm{~ms}$ ) do ramo direito ou esquerdo do feixe de His foram, arbitrariamente, excluídos da análise no domínio do tempo.

Para análise estatística, empregamos o teste $t$ de Student para comparação de médias, teste exato de Fisher para comparação de proporções e fórmulas padrões para determinação de sensibilidade e especificidade. Consideramos estatisticamente significante erro alfa $\leq 5 \%$.

\section{Resultados}

Dos 27 pacientes com TV, cinco apresentavam bloqueio completo de ramo (QRS $\geq 120 \mathrm{~ms})$ e foram excluídos da análise no DT, sendo que quatro eram portadores de bloqueio de ramo direito (BRD) e um de ramo esquerdo (BRE). Nos 22 pacientes restantes, o ECGAR no DT identificou PT em $16(72,7 \%)$, sendo 15 com três variáveis positivas e um com duas variáveis positivas. Nos 30 indivíduos do grupo controle, PTs foram demonstrados em quatro $(5,6 \%)$ casos sendo dois com apenas duas variáveis positivas.
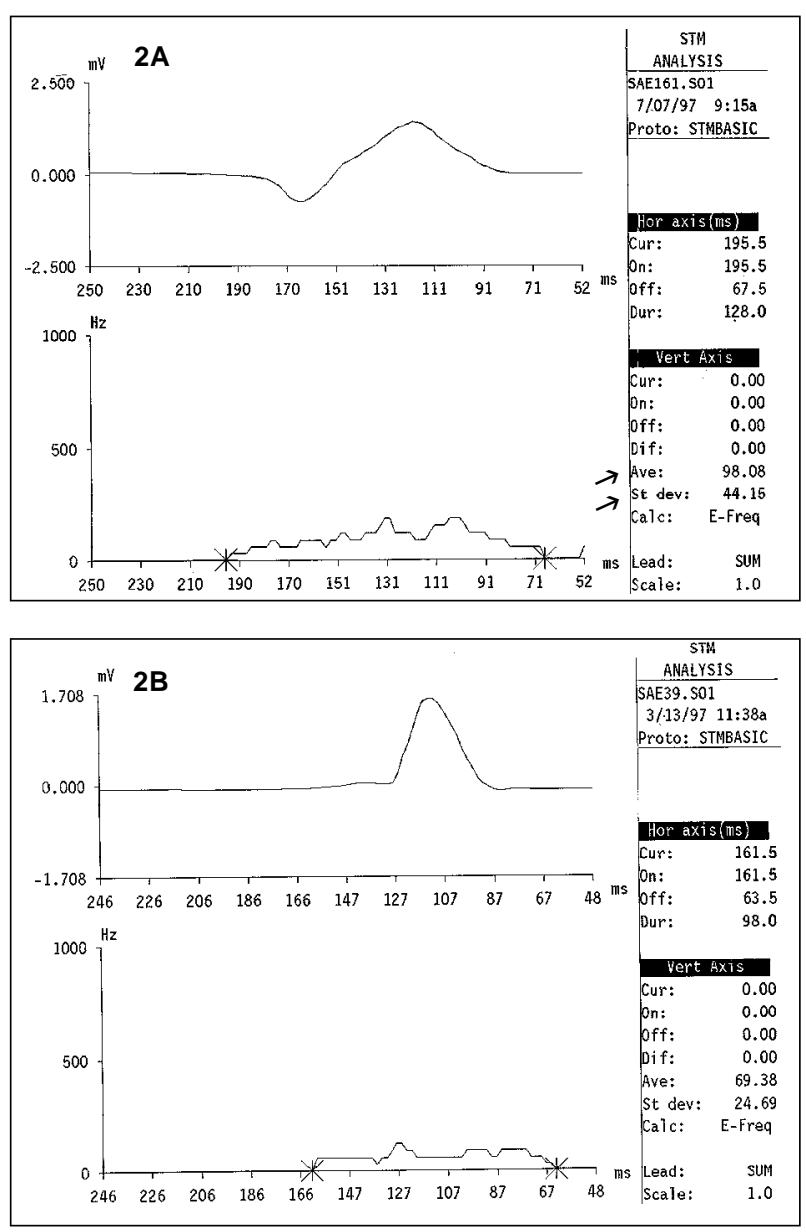

Fig. 2 - ECGAR no domínio da freqüência: banda delimitadora da energia da frequiência do sinal (BD), média (Ave) e desvio padrão (Std) de pacientes com TV (fig. 2A) e de pacientes do grupo controle (fig. 2B). Em 2A, os valores da BD (seta) estão fora dos limites da normalidade.

No DF, as quatro variáveis analisadas nos grupos $\mathrm{TV}$, controle e normal foram respectivamente: 1$) \mathrm{CEM}=$ $94,5 \pm 2,1 ; 96,4 \pm 0,72(\mathrm{p}=0,02)$ e $96,1 \pm 0,68 ; 2) \mathrm{CEDP}=$ $7,8 \pm 3,6 ; 4,8 \pm 1,1(\mathrm{p}=0,05)$ e $4,7 \pm 0,9 ; 3) \mathrm{BDM}=86,4 \pm 17,2$; $71,1 \pm 4,1(\mathrm{p}=0,04)$ e $68,1 \pm 4,6 \mathrm{e} 4) \mathrm{BDDP}=34,2 \pm 13,3 ; 23,9 \pm 3,9$ $(\mathrm{p}=0,05)$ e $23,3 \pm 3,7$. Os pacientes tiveram portanto menores valores de CEM e maiores de CEDP, BDM e BDDP em comparação aos controles.

Os valores de anormalidade foram definidos a partir do intervalo de confiança, com erro alfa $<10 \%$, das médias do grupo normal e consistiram em (valores anormais): CEM $<95, \mathrm{CEDP}>6, \mathrm{BDM}>78 \mathrm{e}$ BDDP $>31$. Foi arbitrariamente padronizado que o encontro de duas variáveis quaisquer anormais tornavam o ECGAR positivo no $\mathrm{DF}$, indicando a presença de distúrbio da condução intra-mural. Com esta padronização os índices de sensibilidade e especificidade foram determinados, sendo que a sensibilidade foi calculada, no grupo TV, separadamente de acordo com a exclusão e inclusão dos casos com bloqueio de ramo. A tabela II compara entre os DTs, DFs e da análise combinada os valores de sensibilidade e especificidade para todos os casos estudados. Nos pacientes com QRS estreito ( $<120 \mathrm{~ms}$ ) a análise em 


\begin{tabular}{|c|c|c|c|}
\hline Grupo/ $\mathrm{N}^{\circ}$ pacientes & $\mathrm{TV} / 27$ & Controle/30 & \\
\hline Idade & $55 \pm 8$ & $52 \pm 9$ & $\mathrm{p}=0,19 \mathrm{NS}$ \\
\hline Sexo masculino & $20(74,0 \%)$ & $22(73,0 \%)$ & $\mathrm{p}=0,94 \mathrm{NS}$ \\
\hline Doença arterial coronária & $17(63,0 \%)$ & $18(60,0 \%)$ & $\mathrm{p}=0,81 \mathrm{NS}$ \\
\hline Infarto do miocárdio prévio & $13(48,0 \%)$ & $15(50,0 \%)$ & $\mathrm{p}=0,88 \mathrm{NS}$ \\
\hline Cardiomiopatia chagásica & $3(11,0 \%)$ & $4(13,0 \%)$ & $\mathrm{p}=0,86 \mathrm{NS}$ \\
\hline Cardiomiopatia hipertrófica & $2(7,4 \%)$ & $2(6,6 \%)$ & $\mathrm{p}=0,69 \mathrm{NS}$ \\
\hline DAVD & $2(7,4 \%)$ & $1(3,3 \%)$ & $\mathrm{p}=0,85 \mathrm{NS}$ \\
\hline Cardiomiopatia dilatada & $3(11,0 \%)$ & $5(16,6 \%)$ & $\mathrm{p}=0,76 \mathrm{NS}$ \\
\hline \multicolumn{4}{|c|}{ Classe funcional para insuficiência cardíaca da NYHA } \\
\hline I & $12(44,4 \%)$ & $14(46,7 \%)$ & $\mathrm{p}=0,87 \mathrm{NS}$ \\
\hline II & $11(40,7 \%)$ & $11(36,6 \%)$ & $\mathrm{p}=0,75 \mathrm{NS}$ \\
\hline III & $4(14,8 \%) 5(16,7 \%)$ & $\mathrm{p}=0,86 \mathrm{NS}$ & \\
\hline IV & $0(0,0 \%)$ & $0(0,0 \%)$ & - \\
\hline Bloqueio de ramo & $5(18,5 \%)$ & $5(16,6 \%)$ & $\mathrm{p}=0,86 \mathrm{NS}$ \\
\hline
\end{tabular}

\begin{tabular}{|c|c|c|c|c|c|c|}
\hline \multirow[t]{2}{*}{ Bloqueio de ramo } & \multicolumn{2}{|c|}{ Domínio do tempo } & \multicolumn{2}{|c|}{ Domínio da freqüência } & \multicolumn{2}{|c|}{ Análise combinada } \\
\hline & $\begin{array}{l}\text { Sem } \\
\text { bloqueio de } \\
\text { ramo }\end{array}$ & $\begin{array}{c}\text { Com } \\
\text { bloqueio de } \\
\text { ramo }\end{array}$ & $\begin{array}{l}\text { Sem } \\
\text { bloqueio de } \\
\text { ramo }\end{array}$ & $\begin{array}{l}\text { Com } \\
\text { bloqueio de } \\
\text { ramo }\end{array}$ & $\begin{array}{l}\text { Sem } \\
\text { bloqueio de } \\
\text { ramo }\end{array}$ & $\begin{array}{l}\text { Com } \\
\text { bloqueio de } \\
\text { ramo }\end{array}$ \\
\hline Sensibilidade & $72,7 \%$ & $59,3 \%$ & $59,1 \%$ & $63,0 \%$ & $81,8 \%$ & $81,5 \%$ \\
\hline Especificidade & $84,0 \%$ & $86,7 \%$ & $84,0 \%$ & $86,7 \%$ & $84,0 \%$ & $86,7 \%$ \\
\hline Grupos & TV/Cont & TV/Cont & TV/Cont & TV/Cont & TV/Cont & TV/Cont \\
\hline Exame positivo & $16 / 4$ & $16 / 4$ & $13 / 4$ & $17 / 4$ & $18 / 4$ & $22 / 4$ \\
\hline Exame negativo & $6 / 21$ & $11 / 26$ & $9 / 21$ & $10 / 26$ & $4 / 21$ & $5 / 26$ \\
\hline positivo & $80,0 \%$ & $80,0 \%$ & $76,5 \%$ & $81,0 \%$ & $81,8 \%$ & $84,6 \%$ \\
\hline
\end{tabular}

DT apresentou maior sensibilidade que em DF $(72,7 \%$ versus $59,1 \% \mathrm{p}=\mathrm{ns}$ ). Dos 22 pacientes com TV e sem bloqueio de ramo, dois apresentaram DT negativo e DF positivo e cinco com DT positivo apresentaram DF negativo. Incluindo os pacientes com bloqueio de ramo, a sensibilidade do DT foi reduzida para 59,3\% e a do DF foi elevada para $63 \%$ (dos cinco casos com bloqueio, quatro apresentaram DF positivo). A especificidade foi elevada para os dois tipos de análise, com $86,7 \%$ tanto para o DT como para o DF. A análise combinada DTe DF apresentou tendência para maior acurácia diagnóstica, identificando corretamente 22 dos 27 pacientes com taquicardia ventricular (sensibilidade de $81,5 \%$ e especificidade de $86,7 \%$ ) em relação à análise isoladanoDT ( $\mathrm{p}=0,07 ; \mathrm{ns})$.

\section{Discussão}

A análise pelo ECGAR no DF tem sido objeto de vários estudos sobre a atividade elétrica ventricular e risco para desenvolvimento de TV, com alguns demonstrando maior acurácia diagnóstica com análise no $\mathrm{DT}^{9,10,12}$, outros com análise no $\mathrm{DF}^{11,13,14}$ e outros com análise combinada de ambos as técnicas ${ }^{4,5}$.

Kelen e $\mathrm{col}^{13}$ desenvolveram método capaz de detectar a variação abrupta e freqüente da energia do sinal ao lon- go da atividade ventricular, através do emprego do coeficiente de correlação de Pearson, denominando os resultados adversos de turbulência espectral. Obtiveram com esta técnica sensibilidade de $88 \%$ e especificidade de $100 \%$ para identificação de TV induzida, com acurácia preditiva de 94\% para DF e 73\% para DT. Recentemente, este estudo teve suas conclusões corroboradas por Copie e $\mathrm{col}^{14}$.

Os resultados adversos encontrados na literatura sobre a eficácia do DF podem ser explicados, em parte, pela diversidade de métodos utilizados, sendo que a maioria dos estudos ocorreu em pacientes com doença coronária e IM prévio. Machac e $\mathrm{col}^{12}$ encontraram em pacientes com e sem TV sustentada, submetidos ao ECGAR, sensibilidade de $85 \%$ e $77 \%$ para os DTs e DFs, respectivamente, com especificidade de $78 \%$ em ambos os domínios. Odemuyima e $\mathrm{col}^{7}$, em outro estudo, concluíram que o DT apresentou sensibilidade de $70 \%$ para detecção de TV e foi superior ao DF em prever eventos arrítmicos após IM.

Em pacientes com cardiomiopatia dilatada, Grimm e col ${ }^{15}$, utilizando um grupo controle de indivíduos saudáveis, encontraram na identificação de TV, sensibilidade de $35 \%$ e $18 \%$ e especificidade de $80 \%$ e $94 \%$ para os DT e DF respectivamente.

Bonato e $\mathrm{col}^{16}$, estudando pacientes com DAVD e empregando análise combinada DT e DF, encontraram po- 
tenciais fragmentados em $88 \%$ dos pacientes com TV sustentada clínica e em $64 \%$ dos pacientes sem essa arritmia, denotando uma baixa especificidade do método.

Na presença de cardiomiopatia hipertrófica, um estudo ${ }^{17}$ demonstrou superioridade do DF em relação ao DT na identificação de TV não sustentada (sensibilidade de 95\%), mas ambos os métodos não foram úteis no reconhecimento do risco de morte súbita.

Em nosso estudo, a análise isolada no DF não apresentou vantagens em relação ao DT, sendo que em pacientes com QRS estreito ( $<120 \mathrm{~ms}$ ) oDT, embora sem significância estatística, apresentou sensibilidade maior que o DF $(72,7 \%$ versus 59,1\%). A análise combinada de ambos os domínios aumentou a sensibilidade para todos os pacientes de 59,3\% (DT) para 81,5\% (DT+DF), mantendo a mesma especificidade de $86,7 \%$. Este aumento ocorreu mesmo excluindo-se os casos com bloqueio de ramo.
A utilização, em nosso estudo, de um grupo de indivíduos normais foi necessária para que pudéssemos instituir os valores de normalidade dos parâmetros do DF através de um programa (Predictor II $_{c}$ ) relativamente novo e ainda sem critérios definidos de seus índices.

A análise no DF em pacientes com bloqueio de ramo tem sido amplamente aceita, por outro lado, o uso do DT não tem sido recomendável, em especial, nos casos de BRD ${ }^{13,18,19}$. Dos cinco casos de bloqueio de ramo em pacientes com TV, quatro apresentavam BRD (dois com cardiopatia chagásica, um com DAVD e um com IM prévio) motivo, pelo qual, excluímos os pacientes com QRS largo da análise no DT.

Os resultados nos levam a acreditar que ambas as análises nos domínios do tempo e freqüência são úteis em identificar pacientes com TV monomórfica sustentada clínica e que a análise combinada destes domínios aumenta a acurácia diagnóstica a despeito da presença de bloqueio de ramo.

\section{Referências}

1. Simson MB - Use of signals in the terminal QRS complex to identify patients with ventricular tachycardia after myocardial infarction. Circulation 1981; 64: 235-42.

2. Buckingham TA, Ghosh S, Homan SM et al - Independent value of signal averaged electrocardiography in identify patients with ventricular tachycardia with coronary artery disease. Am J Cardiol 1987; 59: 568-72.

3. Vester EG, Strauer BE - Ventricular late potencials: state of the art and future perspectives. Eur Heart J 1994; 15(suppl C(LA-): 34-48.

4. Ahuja RK, Turitto G, Ibrahim B - Combined time-domain and spectral turbulence analysis of the signal-averaged ECG improves its predictive accuracy in postinfarction patients. J Electrocardiol 1994; 27 (suppl (LA-): 202-6.

5. Gramatikov BI - Detection of late potencials in the signal-averaged ECG combining time and frequency domain analysis. Med Biol Eng Comput 1993; 31: 333-9.

6. Kulakowski P, Malik M, Poloniecki J et al - Frequency versus time domain analysis of signal-averaged electrocardiograms. Identification of patients with ventricular tachycardia after myocardial infarction. J Am Coll Cardiol 1992; 20: 135-43.

7. Odemuyiwa O, Malik M, Poloniecki J et al - Frequency versus time domain analysis of signal-averaged electrocardiograms. Stratification of postinfarction patients for arrhythmic events. J Am Coll Cardiol 1992; 20: 144-50.

8. Kuchar DL, Thorburn CW, Samuel NL - Prediction of serious arrhythmic events after myocardial infarction: signal-averaged electrocardiogram, Holter monitoring and radionuclide ventriculography. J Am Coll Cardiol 1987; 9: 531-8.

9. Nogami A, Lesaka Y, Akiyama J et al-Combined use of time and frequency domain variables in signal-averaged ECG as a predictor of inducible sustained monomorphic ventricular tachycardia in myocardial infarction. Circulation 1992; 86: 780-9

10. Cain ME, Lindsay BD, Arthur RM, Markham J, Ambos HD - Noninvasive detection of patients prone to life-threatening ventricular arrhythmia by frequency analysis of electrocardiographic signals. In: Zipes DP, Jalife J, eds Cardiac Electrophysiology From Cell to Bedside. Philadelphia: WB Saunders, 1990: 817-31.
11. Worley SJ, Mark DB, Smith WMet al-Comparison of time domain and frequency domain variables from the signal-averaged electrocardiogram: a multivariate analysis. J Am Coll Cardiol 1988; 11: 1041-51

12. Marchac J, Weiss A, Winters SL, Barecca P, Gomes JA - A comparative study of frequency domain and time domain analysis of signal-averaged electrocardiograms in patients with ventricular tachycardia. J Am Coll Cardiol 1988; 11 : 284-96.

13. Kelen GJ, Henkin R, Starr AM, Caref EB, Bloomfield D, El-Sherif N - Spectral turbulence analysis of the signal-averaged electrocardiogram and its predictive accuracy for inducible sustained monomorphic ventricular tachycardia. Am J Cardiol 1991; 67: 965-75.

14. Copie X, Hnatrova K, Staunton A, Camm AJ, Malik M-Spectral turbulence versus time domain analysis of the signal-averaged ECG for risk stratification after myocardial infarction. J Am Coll Cardol 1996; 27(suppl A): 171A.

15. Grimm W, Hoffmann J, KnopU, Winzenburg J, Menz V, Maisch B - Value of time and frequency domain analysis of signal-averaged electrocardiography for arrhythmia risk prediction in idiopathic dilated cardiomyopathy. Pacing Clin Electrophysiol 1996; 19: 1923-7.

16. Bonato P, Bettini R, Speranza G, Furlanello F, Antolini R - Improved late potential analysis in frequency domain. Med Eng Phys 1995; 17: 232-8.

17. Kulakowski P, Counihan PJ, Camm AJ, Mckenna WJ - The value of time and frequency domain, and spectral temporal mapping analysis of the signalaveraged electrocardiogram in identification of patients with hypertrophic cardiomyopathy at increased risk of sudden death. Eur Heart J 1993; 14: 941-50.

18. Lindsay BD, Markham J, Schetman KB, Ambos HD, Caim ME - Identification of patients with sustained ventricular tachycardia by frequency analysis of signalaveraged electrocardiograms despite the presence of bundle branch block. Circulation 1988; 77: 122-30.

19. Buckingham TA, Thessen CM, Stevens LL, Redd RM, Kennedy HL - The effect of conduction defects on the signal-averaged electrocardiographic determination of late potentials. Am J Cardiol 1988; 61: 1265-71. 